\title{
Paranasal Sinus Olfactory Neuroblastoma
}

National Cancer Institute

\section{Source}

National Cancer Institute. Paranasal Sinus Olfactory Neuroblastoma. NCI Thesaurus.

Code C6016.

An olfactory neuroblastoma arising in the paranasal sinus. 